\title{
Nucleotide Analog Chemotherapy
}

National Cancer Institute

\section{Source}

National Cancer Institute. Nucleotide Analog Chemotherapy. NCI Thesaurus. Code C15956.

Use of nucleotide analogs in cancer treatment. 